\title{
Variáveis climáticas influenciam a riqueza, composição e distribuição de plantas exóticas invasoras?
}

Climatic variables influence the richness, composition and distribution of exotic invasive plants?

\author{
T. S. Almeida ${ }^{1}$; R. P. S. Almeida ${ }^{2 *}$; J. R. Fabricante ${ }^{1}$ \\ ${ }^{1}$ Departamento de Biociências, Laboratório de Ecologia e Conservação da Biodiversidade, Universidade Federal de \\ Sergipe, 49500-000, Itabaiana-Sergipe, Brasil \\ ${ }^{2}$ Coordenação de Ciências da Terra e Ecologia, Museu Paraense Emílio Goeldi, 66077-830, Belém-Pará, Brasil \\ *rony_peterson@hotmail.com \\ (Recebido em 7 de setembro de 2020; aceito em 26 de julho de 2021)
}

\begin{abstract}
Neste trabalho buscamos avaliar a influência das variáveis climáticas (precipitação e temperatura) na distribuição de plantas exóticas invasoras em Sergipe, Brasil. Para isso, percorremos $550 \mathrm{~km}$ de rodovias do Estado registrando as plantas exóticas invasoras e relacionamos posteriormente com variáveis climáticas disponíveis em base de dados. No total foram amostradas 46 espécies distribuídas em 17 famílias. Nossos resultados demonstram que a comunidade é influenciada pelas variáveis climáticas testadas. A maior riqueza de espécies ocorreu na zona semiárida de Sergipe. A riqueza foi influenciada principalmente pela precipitação anual, apresentando uma relação negativa. A composição de espécies, por sua vez, foi afetada por quatro variáveis (precipitação anual, sazonalidade da precipitação, temperatura máxima no mês mais quente e temperatura média anual) que determinam suas distribuições. Algumas exóticas invasoras agressivas foram limitadas principalmente pela precipitação anual e sazonalidade de precipitação. No entanto, outras apresentaram alta amplitude de ocorrência, podendo assim se dispersar por diversas regiões já que não apresentam limitações perante as condições climáticas. Assim, concluímos que as condições climáticas podem influenciar de forma positiva ou negativa a composição, riqueza e distribuição de espécies exóticas invasoras no Estado. Nossos resultados auxiliam na compreensão dos efeitos das condições do ambiente na distribuição de espécies exóticas invasoras.
\end{abstract}

Palavras-chave: invasão biológica, espécies alóctones, clima.

In this work we seek to evaluate the influence of climatic variables (precipitation and temperature) on the distribution of invasive alien plants in Sergipe, Brazil. To do this, we traveled $550 \mathrm{~km}$ of state highways recording invasive exotic plants and then relating them to climate variables available in database. In total, 46 species distributed in 17 families were sampled. Our results demonstrate that community is influenced by the tested climatic variables. The greatest species richness occurred in the semiarid zone of Sergipe. Richness was mainly influenced by annual precipitation, showing a negative relationship. Species composition, in turn, was affected by four variables (annual precipitation, seasonality of precipitation, maximum temperature in the hottest month and mean annual temperature) that determine their distributions. Some aggressive invaders were limited mainly by annual rainfall and rainfall seasonality. However, others had a high range of occurrence, thus being able to spread over several regions as they do not have limitations due to climatic conditions. Thus, we conclude that climatic conditions can positively or negatively influence the composition, richness and distribution of invasive alien species in the state. Our results help to understand the effects of environmental conditions on the distribution of invasive alien species.

Keywords: biological invasion, alien species, climate.

\section{INTRODUÇÃO}

A invasão biológica (IB) ocorre quando uma espécie é introduzida fora da sua área de ocorrência natural e passa a se reproduzir, dispersar e causar danos à diversidade desta comunidade da nova área [1,2]. As IBs são consideradas uma das principais ameaças à biodiversidade mundial $[2,3]$ e as espécies conhecidas como exóticas invasoras agressivas ou "transformers", são as principais responsáveis pelos impactos atribuídos a essas IBs [4, 5]. Tais espécies possuem a capacidade de se dispersar, estabelecer e formar massivos populacionais [6], alterando a composição e reduzindo a riqueza e diversidade de espécies nativas [7, 8]. 
As IBs estão diretamente ligadas ao crescimento da população humana, uma vez que o homem é o responsável pela introdução intencional ou acidental de espécies exóticas $[9,10]$. No Brasil, a maior parte das plantas exóticas invasoras foi introduzida de forma intencional, principalmente para a formação de pastagens e exploração agrícola [11]. No entanto, a relação entre as invasões biológicas e a ação do homem não se limita à introdução, pois existe uma correlação positiva entre o crescimento das atividades econômicas e a riqueza de espécies exóticas invasoras [12], de modo que áreas antropizadas ou degradadas são mais suscetíveis a este problema [2]. Os ambientes próximos as rodovias, por exemplo, apresentam condições ótimas para muitas espécies exóticas invasoras, como alta luminosidade, baixa riqueza de espécies, além de facilitar/auxiliar a dispersão das exóticas invasoras [13, 14].

Vários fatores ecológicos também influenciam no estabelecimento de processos de IB $[2,9$, 11]. O clima talvez seja um dos mais relevantes uma vez que é considerado um fator limitante para a composição, distribuição e comportamento das espécies exóticas [15-17]. Condições climáticas como períodos de seca ou de chuvas intensas podem também gerar stress na comunidade nativa ou disponibilizar recursos que geram oportunidades de invasão [18, 19], ou seja, melhor desempenho competitivo das plantas invasoras. Vários estudos demonstram que as variáveis climáticas influenciam a comunidade de plantas exóticas invasoras [17, 20, 21]. A riqueza de espécies exóticas invasoras tende a crescer conforme há aumento da temperatura e decréscimo da precipitação [20,21], aspecto que torna as regiões semiáridas e áridas mais suscetíveis às IB [22].

Apesar do crescente número de estudos sobre as IBs, alguns aspectos sobre o tema ainda são pouco compreendidos, como por exemplo a influência do clima sobre a distribuição de espécies exóticas invasoras nos trópicos. Devido suas características, Sergipe surge como uma área ideal para este tipo de estudo de caso sobre o aspecto levantado. O estado possui clima tropical úmido no litoral chegando rapidamente ao semiárido no interior do continente [23, 24]. Em Sergipe, os poucos e ainda recentes estudos sobre invasão biológica tratam-se de listas florísticas, avaliações da estrutura e impactos sobre a vegetação nativa [6, 8, 25, 26], de modo que nenhum destes estudos investigou a relação do clima com as plantas exóticas invasoras.

Assim, o objetivo desse trabalho foi avaliar como as variáveis bioclimáticas de precipitação e temperatura influenciam na distribuição de plantas exóticas invasoras no estado de Sergipe. Com nosso trabalho, buscamos responder três perguntas: i) As variáveis climáticas influenciam a riqueza de espécies de plantas exóticas invasoras?; ii) As variáveis climáticas podem influenciar a composição de plantas exóticas invasoras?; e iii) existem plantas exóticas invasoras com limiares de ocorrência baseados em variáveis climáticas?

\section{MATERIAL E MÉTODOS}

\section{1 Área de Estudo}

Para responder nossas perguntas, nossa área de estudo foram as rodovias estaduais e federais do estado de Sergipe, sendo elas: SE-100, SE-170, SE-175, SE-230, SE-270 e BR-101, BR-235 (Figura 1). Essas rodovias foram selecionadas por contemplar todos os sentidos (Norte-Sul e Leste-Oeste) e condições climáticas existentes no estado. Foram percorridos no total $550 \mathrm{~km}$ de rodovias, divididos em 55 sessões de $10 \mathrm{~km}$ cada. 


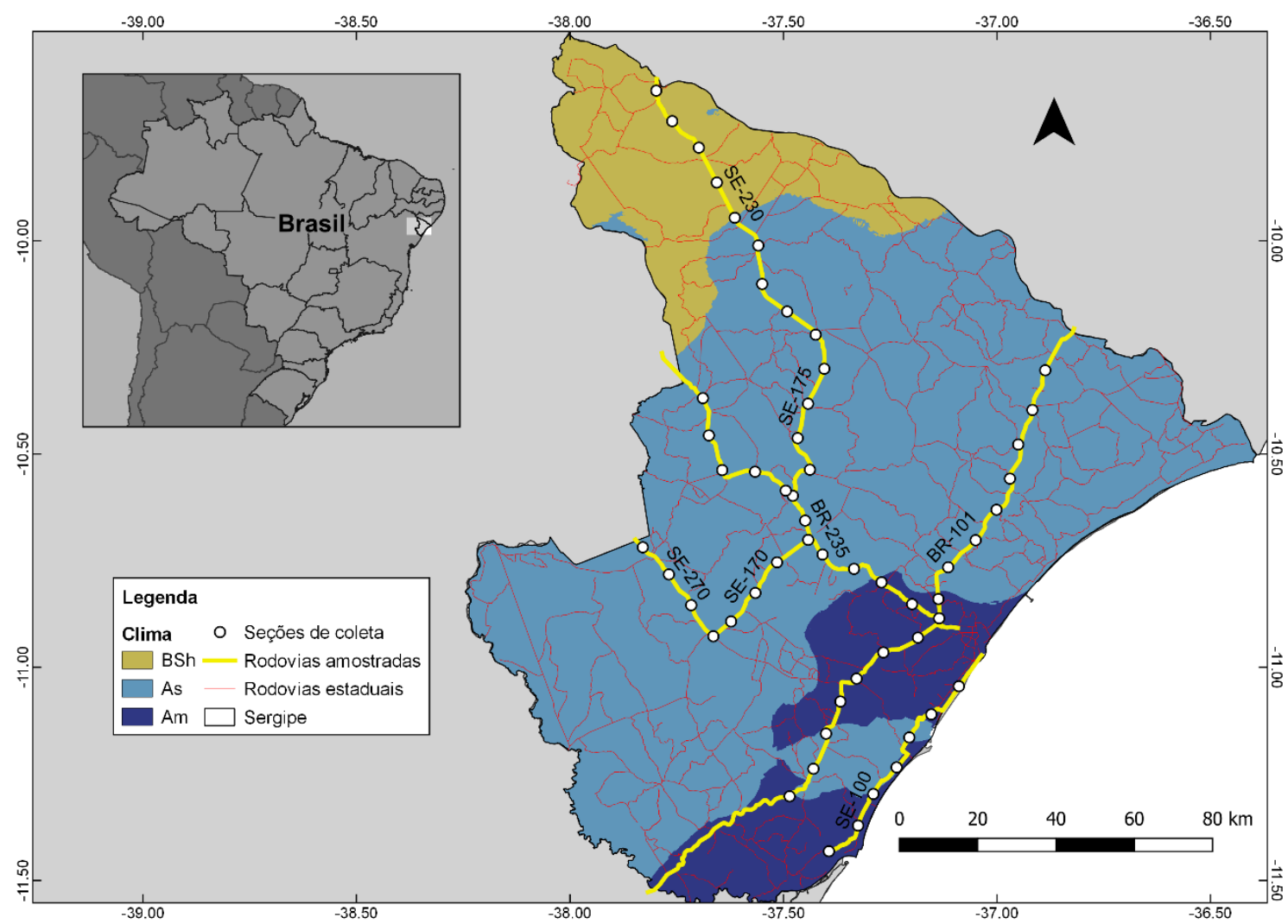

Figura 1: Mapa do estado de Sergipe com destaque para as rodovias utilizadas para coleta de dados da distribuição de plantas exóticas invasoras (SE-100, SE-170, SE-175, SE-270, BR-101, BR-235). Os pontos brancos representam as coordenadas centrais de cada sessão amostrada. (Clima segundo Alvares et al. (2013) [24]: BSh = Semiárido; As = Tropical com verão seco; Am = Tropical de monções).

A utilização de rodovias como local de coleta apresenta vantagens como a possibilidade de aumentar o esforço amostral ao obter dados de distribuição das espécies em uma área maior em menor tempo [13, 14]. Além disso, os ambientes adjacentes as rodovias oferecem condições locais como alta luminosidade, disponibilidade de recursos e impacto antrópico constante que propiciam o estabelecimento e dispersão das plantas exóticas invasoras [13, 14]. Desta forma, estas características facilitam o registro e estudos dos efeitos abióticos com influência regional como o clima [27].

\subsection{Amostragem de Espécies}

A amostragem das espécies foi feita ao longo de seções contínuas de $10 \mathrm{~km}$ nas rodovias, sem intervalo entre uma seção e outra. Os dados foram coletados em expedições realizadas de setembro de 2018 a fevereiro de 2020 . O percurso foi realizado com automóvel a uma velocidade máxima de $60 \mathrm{~km} / \mathrm{h}$. Durante o percurso, todas as plantas exóticas invasoras nos dois lados da rodovia que estavam no limite da visão (aproximadamente 30 metros da borda da pista) eram identificadas e registradas. Este registro ocorreu com o auxílio de duas pessoas, cada uma responsável por um dos lados da rodovia. O procedimento aconteceu em todas as seções. Em caso de dúvidas na identificação da espécie, coletamos material que possibilitasse a identificação posteriormente. Esta metodologia é adaptada de Wilson et al. (1992) [27] e permite avaliar de forma eficiente e ampla a distribuição de espécies ao longo de ambientes.

As espécies não nativas foram identificadas utilizando uma lista prévia das exóticas invasoras do Estado. Esta lista é proveniente de estudos realizados em Sergipe desde 2015 pela equipe do Laboratório de Ecologia e Conservação da Biodiversidade da Universidade Federal de Sergipe, Itabaiana, SE. A identidade taxonômica das exóticas invasoras foi determinada, sempre que possível, no local de registro de forma visual ou, quando necessário, através de registro 
fotográfico utilizando uma câmera Sony Cybershot DSC-H100. Posteriormente, a foto foi comparada com imagens disponíveis em literatura especializada [28-32] ou enviada para identificação por especialistas. Além disso, foi realizada comparação de amostras férteis coletadas com o material depositado no Herbário ASE da Universidade Federal de Sergipe, São Cristóvão, SE.

\subsection{Obtenção das Variáveis Climáticas}

As variáveis climáticas foram obtidas para cada seção utilizando as 19 variáveis bioclimáticas do Worldclim 2 [33] (Tabela S1). Inicialmente importamos o conjunto de dados do site do projeto Worldclim (http://www.worldclim.org/bioclim), no formato GeoTIFF, resolução de 2,5 minutos, série de 1970-2000 e classificação climática de Koppen refinada para o território brasileiro [24]. Posteriormente, os valores das variáveis climáticas para a coordenada de cada seção foram obtidos dos rasters utilizando a função "extract" do pacote raster [34] no software R [35].

\subsection{Análise dos Dados}

Inicialmente, as variáveis climáticas colineares foram identificadas através da correlação de Pearson e retiradas quando apresentaram valores absolutos superiores a $0,7[36,37]$. Entre as dezenove variáveis climáticas testadas, quatro permaneceram nos modelos, sendo elas: 1 temperatura média anual; 2 - temperatura máxima do mês mais quente; 3 - precipitação anual e 4 - sazonalidade de precipitação (Figura S1). Utilizamos sempre as seções como unidades amostrais nas análises de correlação e em todas as análises a seguir.

Para responder a nossa primeira pergunta, utilizamos um modelo linear generalizado (GLM) com distribuição binomial negativa para corrigir a "overdispersion" [38] utilizando a função "glm.nb" do pacote MASS [39]. A riqueza de espécies exóticas invasoras por seção de coleta foi a variável resposta e as quatro variáveis climáticas foram as preditoras. O modelo foi seguido por uma seleção de modelos de AICc [40] utilizando a função "dredge" do pacote MuMIn [41]. Esse método é uma maneira objetiva de avaliar a importância dos modelos mais plausíveis de explicação em relação a todos os modelos candidatos possíveis e tem sido utilizado em trabalhos que apresentam grande número de variáveis [42, 43]. A importância relativa das variáveis foi quantificada pela soma dos pesos de Akaike para cada modelo em que essa variável aparece. Assim, uma variável que aparece em modelos com grandes pesos, receberá um valor importância que tenderá para 1 , de forma que os maiores valores mostram as variáveis mais importantes. $\mathrm{O}$ ponto de corte de 0,6 foi arbitrário e atuou diferenciando variáveis menos importantes das mais importantes [40, 44].

Para a segunda pergunta, avaliamos a relação da composição de plantas exóticas invasoras com as variáveis climáticas através de Análise Multivariada de Permutação (PERMANOVA), utilizando a função "adonis" do pacote vegan [45]. As variáveis foram colocadas por ordem de importância biológica no modelo, segundo a literatura e o conhecimento do especialista $[17,18$, 20]. Utilizamos o índice de Jaccard que considera presença e ausência e 999 aleatorizações [46]. Foi realizada Análise de Redundância (RDA) para visualizar a ordenação utilizando a função "rda" do pacote vegan [45].

Finalmente, para a nossa terceira pergunta, fizemos uma Análise de Limiares para Taxa Indicadores (TITAN) para examinar a influência das variáveis climáticas em limiares de ocorrências das espécies [47]. Utilizamos a função "titan" do pacote TITAN2 [48]. Essa análise indica espécies que apresentam mudança na sua ocorrência ao longo de uma variável contínua, através de valores de IndVal (estimando a associação entre cada táxon com a variável) e escores para valores positivos e negativos de cada ponto de mudança [49]. Apenas espécies com ocorrências $>5$ foram incluídas nas análises, utilizando uma matriz de presença e ausência com 500 aleatorizações [47]. Informações adicionais sobre a utilização dessa análise podem ser obtidas em King et al. (2011) [49]. 
Todas as análises estatísticas foram feitas utilizando o software $\mathrm{R}$ [35] com nível de significância (alfa) de 5\%. As figuras e pranchas foram feitas utilizando os pacotes ggplot2 [50] e gridExtra [51], respectivamente.

\section{RESULTADOS}

Foram registradas 46 espécies pertencentes a 39 gêneros e 17 famílias (Tabela S2). As famílias com mais representantes foram Poaceae com 16 espécies e Fabaceae com cinco. O gênero que apresentou o maior número de espécies foi Cenchrus com quatro táxons, seguido de Cosmos, Crotalaria, Eragrostis e Melinis com duas espécies cada. As espécies com maior número de registros foram Leucaena leucocephala (Lam.) de Wit presente em 51 sessões, Ricinus communis L. em 46, Eucalyptus spp. em 40 e Terminalia catappa L. em 38. As sessões com maior número de espécies apresentaram 21 táxons, nas rodovias SE-175 e SE-270, por outro lado, o menor número de espécies foi registrada na SE-100 com três espécies. Em média, foram registradas 12,45 $\pm 4,48$ (média \pm desvio padrão) espécies por sessão.

\subsection{Riqueza de Plantas Exóticas Invasoras}

Encontramos relação entre a riqueza de exóticas invasoras e duas variáveis climáticas, sendo elas: precipitação anual e sazonalidade da precipitação. Entre os modelos concorrentes, o modelo mais parcimonioso explicando a riqueza de plantas foi formado pela variável precipitação anual, seguido do modelo com as duas variáveis de precipitação (precipitação anual e sazonalidade da precipitação) e a sazonalidade da precipitação isoladamente (Tabela 1). Com relação a importância das variáveis nos melhores modelos, apenas precipitação anual apresentou valor superior a 0,6 (Figura 2A). A riqueza de espécies de plantas invasoras demonstrou uma relação negativa com a precipitação anual (Figura 2B), desta forma, é esperado que áreas semiáridas apresentem riqueza superior de exóticas invasoras quando comparadas com áreas mais úmidas.
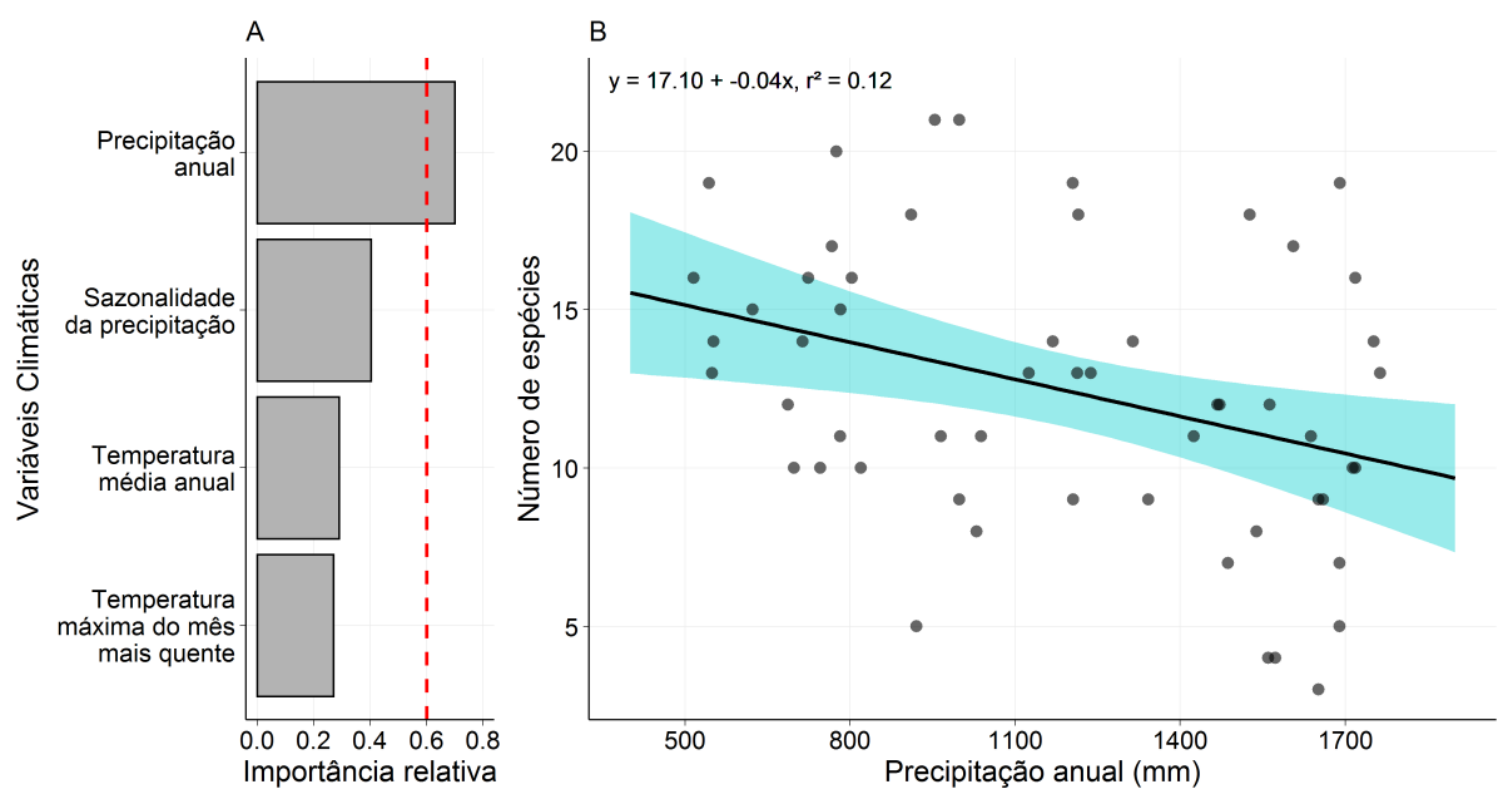

Figura 2: (A) Importância relativa das variáveis climáticas nos melhores modelos e (B) representação do modelo mais parcimonioso selecionado (ver Tabela 1) com a relação entre a riqueza de espécies de plantas exóticas e a precipitação anual. A linha vermelha vertical em A é usada como linha de corte para diferenciar variáveis menos importantes das mais importantes; a faixa azul em B representa o intervalo de confiança da regressão. 
Tabela 1: Resultados dos melhores modelos $(\triangle A I C c<2)$ para os efeitos das variáveis climáticas na riqueza de espécies de plantas exóticas. Os modelos são ranqueados de acordo com o valor de AICc. Ké o número de parâmetros estimados para cada modelo.

\begin{tabular}{cccccc}
\hline Modelos & $\Delta$ AICc & AICc & K & wAICc & R $^{\mathbf{2}}$ \\
\hline Precipitação anual & 0 & 321,78 & 3 & 0,27 & 0,12 \\
Precipitação anual + Sazonalidade da precipitação & 1,68 & 323,46 & 4 & 0,12 & 0,13 \\
Sazonalidade da precipitação & 1,92 & 323,70 & 3 & 0,10 & 0,08 \\
\hline
\end{tabular}

\subsection{Composição de Plantas Exóticas Invasoras}

Encontramos relação entre a composição de plantas exóticas invasoras e a precipitação anual e a sazonalidade da precipitação (Tabela 2), sendo essas as variáveis que modulam a mudança das espécies de plantas exóticas invasoras ao longo das rodovias no estado de Sergipe. A análise de redundância explicou, nos dois primeiros eixos, 14,86\% da variação total dos dados (Tabela S3). As variáveis que mais contribuíram para a formação do eixo 1 foram precipitação anual e sazonalidade da precipitação, ambas relacionadas negativamente com o eixo (Figura 3). Para o eixo 2, as variáveis que mais contribuíram foram a temperatura máxima no mês mais quente e a temperatura média anual, também relacionadas negativamente com o eixo.

Tabela 2: Resultado da PERMANOVA, descrevendo composição da comunidade de plantas exóticas em relação as variáveis climáticas. ( $S S=$ Soma dos quadrados; $M S=$ Média dos quadrados $)$

\begin{tabular}{ccccccc}
\hline Variável & d,f, & SS & MS & Pseudo $\mathbf{F}$ & $\mathbf{R}^{\mathbf{2}}$ & $\boldsymbol{p}^{*}$ \\
\hline Precipitação anual & 1 & 1,73 & 1,73 & 8,55 & 0,13 & $<\mathbf{0 , 0 1}$ \\
Sazonalidade da precipitação & 1 & 0,37 & 0,37 & 1,84 & 0,03 & $\mathbf{0 , 0 3}$ \\
Temperatura máxima do mês mais quente & 1 & 0,34 & 0,34 & 1,68 & 0,03 & 0,06 \\
Temperatura média anual & 1 & 0,33 & 0,33 & 1,66 & 0,03 & 0,06 \\
Resíduo & 50 & 10,11 & 0,20 & & 0,78 & \\
Total & 54 & 12,89 & & & 1 & \\
\hline
\end{tabular}

*valores de p determinados por permutação.

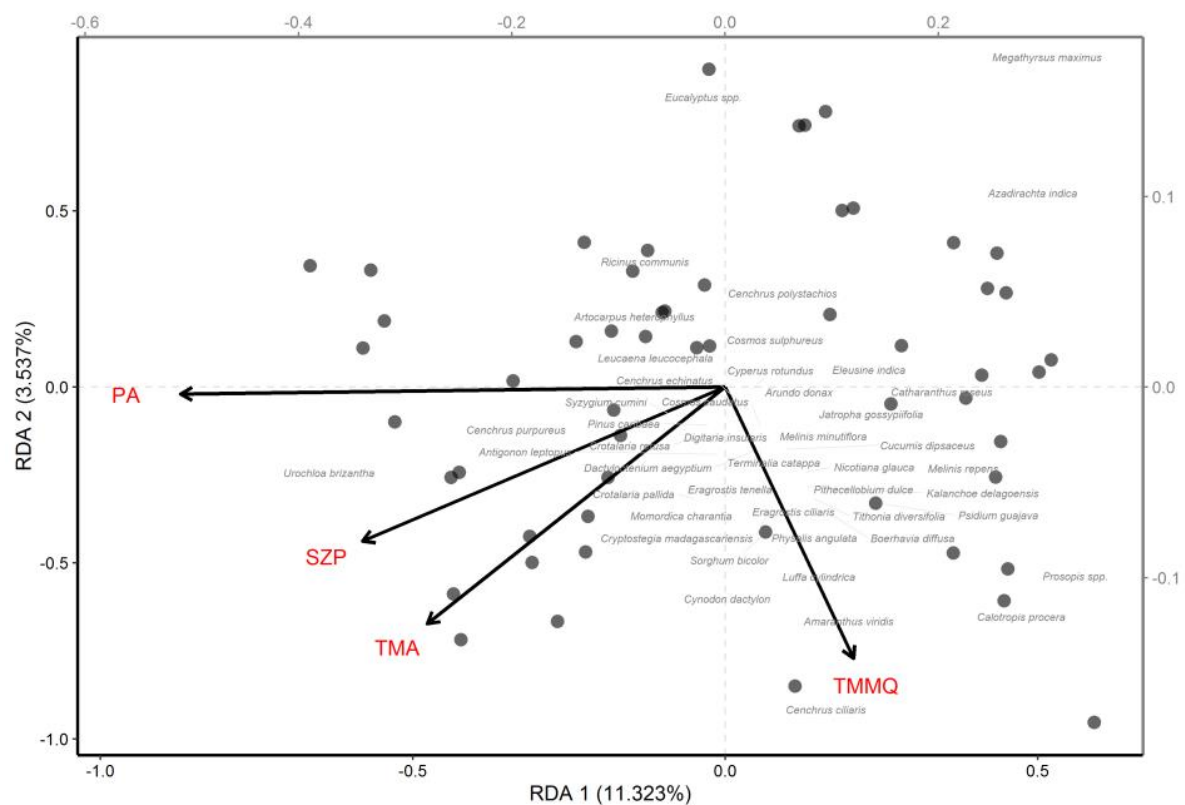

Figura 3: Análise de redundância (RDA) da composição de plantas exóticas com as variáveis climáticas coletadas ao longo das rodovias do estado de Sergipe, Brasil. Os autovalores e explicação

(\%) da variância para os componentes principais estão descritos na Tabela S3 (PA = Precipitação anual; $S Z P=$ Sazonalidade da precipitação; $T M A=$ Temperatura média anual; $T M M Q=$ Temperatura máxima do mês mais quente). 
As espécies amostradas apresentaram limiares de ocorrência por variáveis climáticas. $\mathrm{O}$ TITAN identificou nove espécies $(19,15 \%$ do total) que apresentam mudanças nas suas distribuições em razão do gradiente de pelo menos uma das variáveis climáticas (Figura 4; Tabela S4). Para a precipitação anual, cinco espécies (Melinis repens (Willd.) Zizka, Megathyrsus maximus (Jacq.) B.K.Simon \& S.W.L.Jacobs, Prosopis spp., Calotropis procera (Aiton) W.T. Aiton e Azadirachta indica A. Juss.) foram indicadoras negativas (ou seja, reduzindo sua ocorrência a partir de determinada faixa de aumento da variável) e apenas Urochloa brizantha (Hochst. ex A. Rich.) R.D.Webster foi indicadora positiva (ou seja, reduzindo sua ocorrência a partir de determinada faixa de redução da variável) (Figura 4A). Para sazonalidade de precipitação, cinco espécies (Jatropha gossypiifolia L., Catharanthus roseus (L.) G.Don., M. maximus, A. indica e Prosopis spp.) foram indicadoras negativas e $U$. brizantha foi indicadora positiva (Figura 4B). A temperatura do mês mais quente obteve duas espécies (Eucalyptus spp. e M. maximus) indicadoras negativas e $C$. procera indicadora positiva (Figura 4C). Por último, a temperatura média anual obteve quatro espécies indicadoras negativa (M. maximus, A. indica, Eucalyptus spp. e M. repens) e U. brizantha como indicadora positiva (Figura 4D).
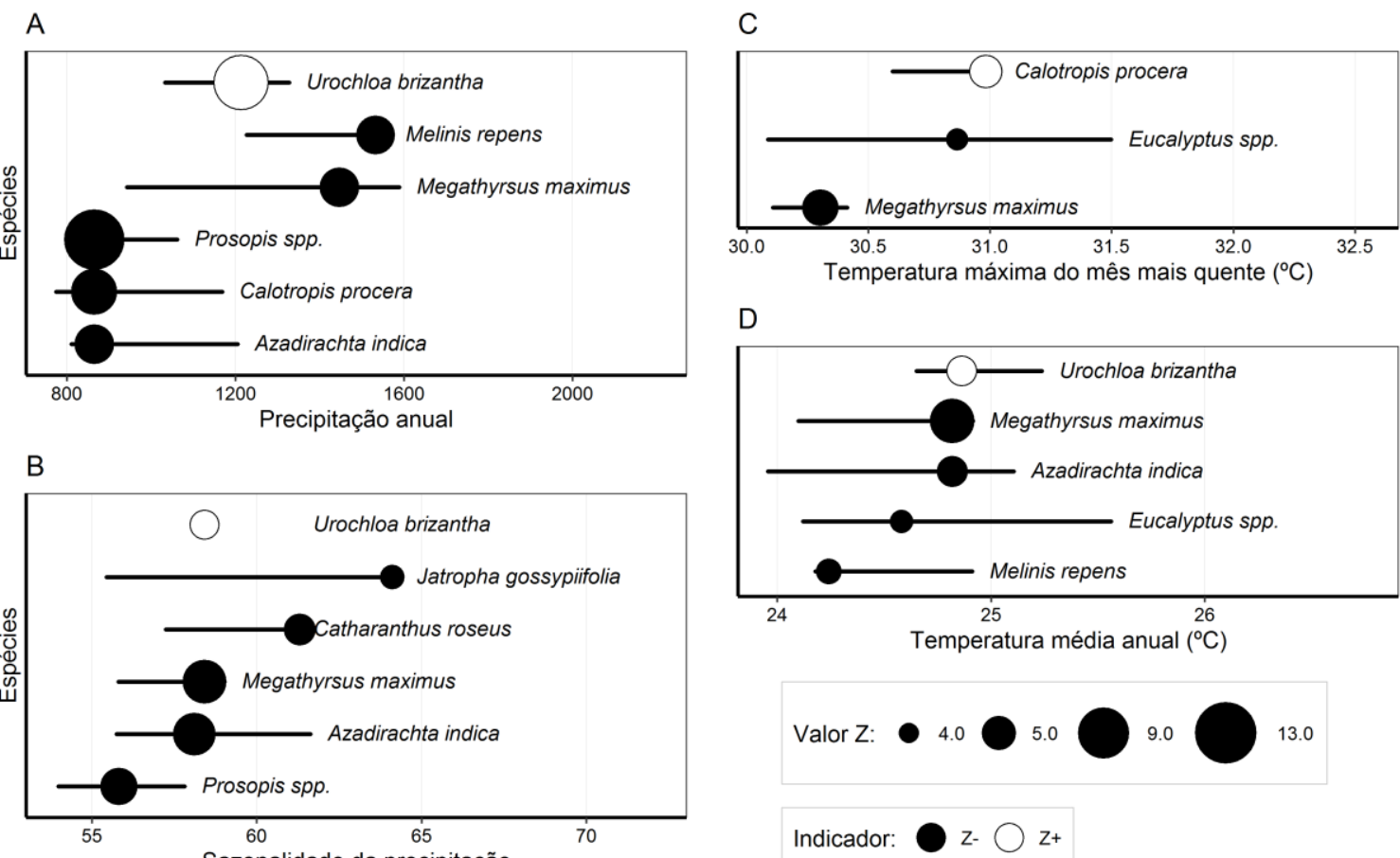

Figura 4: Análise de indicador de limiar (TITAN) para a comunidade de plantas exóticas em resposta a quatro variáveis climáticas: (A) Precipitação anual, (B) Sazonalidade da precipitação, (C) Temperatura máxima do mês mais quente e (D) Temperatura média anual, coletadas ao longo das rodovias do estado de Sergipe, Brasil. As espécies com indicadores puros $(\geq 0,95)$ são plotados em ordem crescente com relação ao ponto de mudança observado. Os símbolos fechados correspondem as espécies de indicadores negativos (z-) enquanto os abertos correspondem as de indicadores positivos ( $z+)$. Os tamanhos dos símbolos são dimensionados em proporção aos escores z. As linhas horizontais pretas representam os percentis 5 e 95 com base nas aleatorizações. Informações complementares dos valores das espécies podem ser encontrados na Tabela S4.

\section{DISCUSSÃO}

Nosso estudo testou de forma pioneira a influência de variáveis climáticas na distribuição de plantas exóticas invasoras no Estado de Sergipe. Demonstramos que a riqueza de espécies possui uma relação negativa com a variável climática precipitação anual, de forma que mais espécies são encontradas em regiões com menor volume de chuvas, a exemplo da região semiárida. Além 
disso, evidenciamos que essa mesma variável e a sazonalidade de precipitação influenciam na composição de exóticas invasoras. Por último, apresentamos espécies que exibem mudanças nas suas distribuições em razão da precipitação anual, sazonalidade de precipitação, temperatura do mês mais quente e temperatura média anual. Assim, geramos informações que podem auxiliar na mitigação do problema e direcionar políticas públicas.

O número de exóticas invasoras encontradas neste trabalho representa $54,02 \%$ das 87 espécies exóticas registradas em Sergipe e 16,20\% das 290 espécies do Nordeste [52]. Estudos realizados no Nordeste também apresentaram número de exóticas invasoras inferiores ao deste trabalho. No Parque Nacional do Catimbau foram registradas 18 espécies [53], em sítios de Caatinga na cidade de Petrolina foram registradas 29 espécies [54] e na Área de Proteção Ambiental Morro do Urubu foram encontradas 37 espécies exóticas invasoras [55]. Esta diferença na quantidade de espécies pode ser justificada pelo tamanho da área amostral e da variação climática dela, além disso, a alta riqueza pode ser justificada pela área de estudo deste trabalho, uma vez que rodovias apresentam condições ideais para o estabelecimento de exóticas invasoras [13, 14].

A predominância das famílias Poaceae e Fabaceae também ocorre em grande escala, uma vez que são as famílias com mais espécies exóticas invasoras no Brasil [11, 56]. Esse fato pode ser explicado pelo potencial econômico dessas famílias, que incluem diversas espécies introduzidas intencionalmente, especialmente para uso na agricultura e pecuária [11] As espécies mais frequentes no presente estudo foram aquelas reconhecidamente agressivas, como L. leucocephala, uma das 100 piores exóticas invasoras do mundo [57], $R$. communis, espécie de difícil controle que ocorre em todo o país [29] e T. catappa, que altera a composição, riqueza e biodiversidade de plantas nativas de ambientes que invade [8]. Desta forma, estas espécies estão adaptadas às diversas condições climáticas que ocorrem em Sergipe [29, 31]

Altos valores de riqueza de plantas exóticas em áreas quentes, secas e com poucas chuvas, como o semiárido, já foram relatados em outros estudos [21, 22]. A sazonalidade, índice de cobertura e fenologia da cobertura vegetal no Nordeste respondem diretamente à sazonalidade de precipitação [58, 59]. Além disso, as altas temperaturas associadas a baixa quantia de chuvas geram stress hídrico na vegetação nativa [19]. Assim, os períodos de seca aumentam a taxa de mortalidade na vegetação nativa gerando nichos vagos que facilitam a propagação de exóticas invasoras naquele local $[18,22]$. Desta forma, é esperado que essas espécies ocupem mais rapidamente os ambientes após eventos de seca, já que possuem vantagem competitiva e rápido crescimento [18, 2]. Por outro lado, a menor riqueza encontrada nas áreas com maior precipitação pode estar ligada à estabilidade da comunidade nativa nos ambientes que apresentam chuvas regulares [18].

Assim como a vegetação nativa da região, a comunidade de exóticas invasoras também é influenciada principalmente pela precipitação $[23,58,59]$. Estas variáveis podem atuar definindo as espécies que conseguem se estabelecer e se manter em uma determinada área. Nas áreas semiáridas, a baixa quantidade de chuvas e a irregularidade na sua distribuição ao longo do ano pode acarretar no desaparecimento de algumas espécies nativas e facilitar o estabelecimento de espécies exóticas [2,60].

Os efeitos das variáveis notados nos outros parâmetros podem ser melhor compreendidos quando observamos as populações e limiares de ocorrência das espécies. Algumas espécies apresentam ocorrência limitadas à menores valores de precipitação, como é o caso de Prosopis spp. e C. procera. Essas espécies demonstraram baixa amplitude de tolerância para os fatores climáticos, estando presentes especialmente em sítios com altas temperaturas e baixa precipitação. Além disso, são reconhecidamente exóticas invasoras agressivas no semiárido e possuem a capacidade de alterar drasticamente a diversidade nas áreas onde ocorrem, afetando as espécies nativas [7, 28, 29].

As gramíneas $C$. ciliaris e $M$. repens foram limitadas de forma semelhante, no entanto, a ocorrência destas espécies não é limitada pelo clima da mesma forma que as duas últimas, uma vez que também são encontradas em áreas mais úmidas, invadindo inclusive áreas em regiões sob os domínios de florestas úmidas [29]. Já $U$. brizantha e M. maximus mostraram ampla ocorrência, ocorrendo junto com as espécies do semiárido e adicionalmente em valores mais altos de precipitação e temperatura. Essas espécies podem representar grande ameaça para a biodiversidade do Estado graças as suas características. Ambas já apresentam comportamento 
invasor na Mata Atlântica do Estado, graças a capacidade de formar populações viáveis em diferentes ambientes, reduzindo a diversidade biológica nestas áreas $[6,26]$.

A maior parte das gramíneas foram introduzidas intencionalmente principalmente para o uso como forragem animal [11]. $\mathrm{O}$ aspecto da introdução intencional em múltiplos ambientes, unido à programas de pesquisa agronômica e melhoramento genético dessas espécies coopera com a rápida adaptação e invasão dessas espécies à múltiplos ambientes [60]. Por outro lado, Eucalyptus spp. é uma espécie naturalizada cujo processo de invasão ainda não foi registrado para o Estado [25]. No entanto, durante a coleta de dados, nas áreas litorâneas do Estado foram observadas pequenas populações de Eucalyptus spp. se formando distante às árvores adultas da mesma espécie, o que demonstra que provavelmente esta espécie está em processo de adaptação e transformação do estado de naturalizada para exótica invasora [4]. Dessa forma, a dispersão dessa espécie pode acarretar futuras invasões e maiores impactos para a biodiversidade já bastante ameaçada. Assim é necessário que o plantio dessa espécie seja evitado visando reduzir sua dispersão, além disso, são necessários estudos para entender os possíveis impactos que ela pode causar à biodiversidade nativa.

O efeito das variáveis ambientais na comunidade local de plantas exóticas invasoras ainda é pouco compreendido e se torna fundamental diante dos efeitos desse grupo. Poucos trabalhos avaliam a fisiologia dessas espécies e o seu potencial de causar danos ou alterar a comunidade nativa. Além disso, é esperado que as mudanças climáticas modifiquem as IBs, ao alterar a amplitude de ocorrência de espécies invasoras, transformar espécies antes naturalizadas em invasoras ou invasoras agressivas em espécies menos importantes [16].

\section{CONCLUSÃO}

Demonstramos que algumas variáveis ambientais influenciam na riqueza e composição de plantas exóticas invasoras em Sergipe. Embora uma maior riqueza de espécies tenha sido encontrada no semiárido, as regiões com precipitação mais abundantes, também apresentaram grande quantidade de espécies exóticas invasoras. Além disso, demonstramos que as espécies que compõem a comunidade de exóticas invasoras muda ao longo do gradiente climático. Destacamos aqui espécies de plantas exóticas as quais necessitam de prioridade no seu controle já que apresentam maiores distribuições ao longo do gradiente climático e são propensas a se alastrarem por não apresentarem restrições de clima, $U$. brizantha e M. maximus. Assim, com informações sobre a distribuição das plantas exóticas no Estado e a relação com as variáveis climáticas podemos entender possíveis ampliações de distribuições das espécies, sobretudo aqueles causados por efeitos das mudanças climáticas.

\section{AGRADECIMENTOS}

Agradecemos à Universidade Federal de Sergipe por disponibilizar os serviços do setor de transporte para auxiliar nas coletas de dados. Agradecemos aos dois revisores anônimos pelas sugestões no manuscrito. RPSA agradece o apoio da Coordenação de Aperfeiçoamento de Pessoal de Nível Superior - Brasil (CAPES) - Código de Financiamento 001.

\section{REFERÊNCIAS BIBLIOGRÁFICAS}

1. Parker IM, Simberloff D, Lonsdale WM, Goodell K, Wonham M, Kareiva PM, et al. Impact: Toward a framework for understanding the ecological effects of invaders. Biol Invasions. 1999 Mar;1(1):3-19, doi: 10.1023/A:1010034312781

2. Ziller SR. Os processos de degradação ambiental originados por plantas exóticas invasoras. Ciência Hoje. 2001;30:1-6.

3. Bellard C, Cassey P, Blackburn TM. Alien species as a driver of recent extinctions. Biol Lett. 2016 Mar;12(4), doi: 10.1098/rsbl.2015.0623 
4. Richardson DM, Pyšek P, Rejmánek M, Barbour MG, Dane Panetta F, West CJ. Naturalization and invasion of alien plants: Concepts and definitions. Divers Distrib. 2000 Dez;6(2):93-107, doi: 10.1046/j.1472-4642.2000.00083.x

5. Moro MF, Souza VC, de Oliveira-Filho AT, de Queiroz LP, de Fraga CN, Rodal MJN, et al. Alienígenas na sala: O que fazer com espécies exóticas em trabalhos de taxonomia, florística e fitossociologia? Acta Bot Brasilica. 2012 Out;26(4):991-999, doi: 10.1590/S0102-33062012000400029

6. Lima RA. Avanços e atualidades na botânica brasileira. Rio Branco (Acre): Stricto Sensu; 2020. Capítulo 9, Estrutura populacional de espécies exóticas invasoras em sítios de Mata Atlântica, Itabaiana, Sergipe, Brasil; p. 126-138, doi: 10.35170/ss.ed.9786586283006.09

7. Andrade LA, Fabricante JR, de Oliveira FX. Biological invasion by Proposis juliflora (Sw.) DC.: Impacts on diversity and structure of the shrubby-arboreal component of caatinga in the state of Rio Grande do Norte, Brazil. Acta Bot Brasilica. 2009;23(4):935-943, doi: 10.1590/s010233062009000400004

8. Santos JPB, Fabricante JR. Population structure and effects by the invasive exotic Indian-Almond over autochthonous vegetation from a sandbank. Neotrop Biol Conserv. 2018 Out-Dez;13(4):295-302, doi: 10.4013/nbc.2018.134.03

9. Mack RN, Simberloff D, Mark LW, Evans H, Clout M, Bazzaz FA. Biotic invasions: causes, epidemiology, global consequences, and control. Ecoll Appl. 2000 Jun;10(3):689-710, doi: 10.1890/1051-0761(2000)010[0689:BICEGC]2.0.CO;2

10. Richardson DM, Rejmánek M. Trees and shrubs as invasive alien species-a global review. Divers Distrib. 2011 Ago;17(5):788-809, doi: 10.1111/j.1472-4642.2011.00782.x

11. Zenni RD. Analysis of introduction history of invasive plants in Brazil reveals patterns of association between biogeographical origin and reason for introduction. Austral Ecol. 2014 Set;39(4):401-407, doi: 10.1111/aec.12097

12. Taylor BW, Irwin RE. Linking economic activities to the distribution of exotic plants. Proc Natl Acad Sci USA. 2004 Dez;101(51):17725-17730, doi: 10.1073/pnas.0405176101

13. Trombulak SC, Frissell CA. Review of ecological effects of roads on terrestrial and aquatic communities. Conserv Biol. 2000 Jan;14(1):18-30, doi: 10.1046/j.1523-1739.2000.99084.x

14. Pauchard A, Alaback PB. Influence of elevation, land use, and landscape context on patterns of alien plant invasions along roadsides in protected areas of south-central Chile. Conserv Biol. 2004 Jan;18(1):238-248, doi: 10.1111/j.1523-1739.2004.00300.x

15. Richardson DM, Bond WJ. Determinants of plant distribution: evidence from pine invasions. Am Nat. 1991 May;137(5):639-668, doi: 10.1086/285186

16. Hellmann JJ, Byers JE, Bierwagen BG, Dukes JS. Five potential consequences of climate change for invasive species. Conserv Biol. 2008 Jun;22(3):534-543, doi: 10.1111/j.1523-1739.2008.00951.X

17. Menuz DR, Kettenring KM. The importance of roads, nutrients, and climate for invasive plant establishment in riparian areas in the northwestern United States. Biol Invasions. 2013 Dez;15(7):16011612, doi: 10.1007/s10530-012-0395-6

18. Davis MA, Grime JP, Thompson K. Fluctuating resources in plant communities: A general theory of invasibility. J Ecol. 2000 Dez;88(3):528-534, doi: 10.1046/j.1365-2745.2000.00473.x

19. Daniel F, Salvador MDA, Silva VBS, Kousky VE. A seca de 2012 no nordeste do Brasil. Climanálise. 2012 Nov;4(November):1-5.

20. Lilley PL, Vellend M. Negative native-exotic diversity relationship in oak savannas explained by human influence and climate. Oikos. 2009 Ago;118(9):1373-1382, doi: 10.1111/j.1600-0706.2009.17503.x

21. Bartomeus I, Sol D, Pino J, Vicente P, Font X. Deconstructing the native-exotic richness relationship in plants. Glob Ecol Biogeogr. 2012 Ago;21(5):524-533, doi: 10.1111/j.1466-8238.2011.00708.x

22. Wilson JR, Foxcroft LC, Geerts S, Hoffman MT, MacFadyen S, Measey J, et al. The role of environmental factors in promoting and limiting biological invasions in South Africa. In: van Wilgen BW, Measey J, Richardson DM, et al. Biological Invasions in South Africa. Cham $(\mathrm{CH})$ : Springer Open; 2020. p. 355-385, doi: 10.1007/978-3-030-32394-3

23. Prata APN, Amaral MCE, Farias MCV, Alves MV. Flora de Sergipe. v. 1. Aracaju (SE): Gráfica e Editora Triunfo; 2013.

24. Alvares CA, Stape JL, Sentelhas PC, De Moraes Gonçalves JL, Sparovek G. Köppen's climate classification map for Brazil. Meteorol Zeitschrift. 2013 Dez;22(6):711-728, doi: 10.1127/09412948/2013/0507

25. Santos JPB, Fabricante JR, Oliveira AM. Espécies exóticas utilizadas na arborização urbana do município de Itabaiana, Sergipe, Brasil. Agroforestalis News. 2018 Jan;3(2):59-71.

26. Araújo KCT, Fabricante JR. Invasão biológica no Parque Nacional Serra de Itabaiana, Sergipe, Brasil. Rev Cien Ambient. 2020 Jul;14(2):43-50, doi: http://dx.doi.org/10.18316/rca.v14i2.6169 
27. Wilson JB, Rapson GL, Sykes MT, Watkins AJ, Williams PA. Distributions and climatic correlations of some exotic species along roadsides in South Island, New Zealand. J Biogeogr. 1992 Fev;19(2):183194, doi: $10.2307 / 2845504$

28. Fabricante JR. Plantas exóticas e exóticas invasoras da Caatinga. v. 1. Florianópolis (SC): Bookess; 2013.

29. Fabricante JR. Plantas exóticas e exóticas invasoras da Caatinga. v. 2. Florianópolis (SC): Bookess; 2013.

30. Fabricante JR. Plantas exóticas e exóticas invasoras da Caatinga. v. 3. Florianópolis (SC): Bookess; 2013.

31. Fabricante JR. Plantas exóticas e exóticas invasoras da Caatinga. v. 4. Florianópolis (SC): Bookess; 2014.

32. Leão TC, Almeida WR, Dechoum MDES, Ziller SR. Espécies exóticas invasoras no Nordeste do Brasil: contextualização, manejo e políticas públicas. Recife (PE): Centro de Pesquisas Ambientais do Nordeste e Instituto Hórus de Desenvolvimento e Conservação Ambiental; 2011.

33. Fick SE, Hijmans RJ. WorldClim 2: new 1-km spatial resolution climate surfaces for global land areas. Int J Climatol. 2017 Mai;37(12):4302-4315, doi: 10.1002/joc.5086

34. Hijmans RJ. raster: Geographic Data Analysis and Modeling. R package version 3.0-12; 2020.

35. R Core Team. R: A language and environment for statistical computing. Vienna (Austria): R Foundation for Statistical Computing; 2020.

36. Dormann CF, Elith J, Bacher S, Buchmann C, Carl G, Carré G, et al. Collinearity: a review of methods to deal with it and a simulation study evaluating their performance. Ecography. 2013 Mai;36(1):27-46, doi: 10.1111/j.1600-0587.2012.07348.x

37. Schober P, Boer C, Schwarte LA. Correlation coefficients: appropriate use and interpretation. Anesth Analg. 2018 Mai;126(5):1763-1768, doi: 10.1213/ANE.0000000000002864

38. Zuur AF, Ieno EN, Elphick CS. A protocol for data exploration to avoid common statistical problems. Methods Ecol Evol. 2010 Fev;1(1):3-14, doi: 10.1111/j.2041-210x.2009.00001.x

39. Venables WN, Ripley BD. Modern Applied Statistics with S. New York (US): Springer; 2002.

40. Guthery FS, Burnham KP, Anderson DR. Model Selection and multimodel inference: a practical information-theoretic approach. J Wildl Manage. 2003;67(3):655, doi: 10.2307/3802723

41. Barton K. MuMIn: Multi-Model Inference. R package version 1.43.17; 2020.

42. Leal IR, Filgueiras BKC, Gomes JP, Iannuzzi L, Andersen AN. Effects of habitat fragmentation on ant richness and functional composition in Brazilian Atlantic Forest. Biodivers Conserv. 2012 Mar;21(7):1687-1701, doi: 10.1007/s10531-012-0271-9

43. Eschtruth AK, Battles JJ. Assessing the relative importance of disturbance, herbivory, diversity, and propagule pressure in exotic plant invasion. Ecol Monogr. 2009 Mai;79(2):265-280, doi: 10.1890/080221.1

44. Everaert G, Deschutter Y, De Troch M, Janssen CR, De Schamphelaere K. Multimodel inference to quantify the relative importance of abiotic factors in the population dynamics of marine zooplankton. $\mathrm{J}$ Mar Syst. 2018 Mai;181:91-98, doi: 10.1016/j.jmarsys.2018.02.009

45. Oksanen J, Blanchet FG, Friendly M, Kindt R, Legendre P, McGlinn D, et al. vegan: Community Ecology Package. R package version 2.5-6, Cran Proj; 2019.

46. Clarke KR. Non-parametric multivariate analyses of changes in community structure. Aust J Ecol. 1993 Mar;18(1):117-143, doi: 10.1111/j.1442-9993.1993.tb00438.x

47. Baker ME, King RS. A new method for detecting and interpreting biodiversity and ecological community thresholds. Methods Ecol Evol. 2010 Fev;1(1):25-37, doi: 10.1111/j.2041210x.2009.00007.x

48. Baker ME, King RS, Kahle D. TITAN2: Threshold Indicator Taxa Analysis. R package version 2.4. Cran Proj; 2019.

49. King RS, Baker ME, Kazyak PF, Weller DE. How novel is too novel? Stream community thresholds at exceptionally low levels of catchment urbanization. Ecol Appl. 2011 Jul;21(5):1659-1678, doi: 10.1890/10-1357.1

50. Wickham H. ggplot2: Elegant Graphics for Data Analysis. J R Stat Soc Ser A (Statistics Soc). 2011;174(1):245-246, doi: 10.1111/j.1467-985x.2010.00676_9.x

51. Auguie B. gridExtra: functions in Grid graphics. R Package Version 2.3. Cran Proj; 2017.

52. Flora do Brasil 2020. Jardim Botânico do Rio de Janeiro [Internet]; 2020 [acesso em 14 mai 2020]. Disponível em: http://floradobrasil.jbrj.gov.br/

53. Silva FO, Fabricante JR. Invasão biológica no Parque Nacional Do Catimbau, Pernambuco, Brasil. Rev Ciências Ambient. 2019 Ago;13(2):17-26, doi: 10.18316/rca.v13i2.5576 
54. Alves JS, Fabricante JR. Exotic invasive flora evaluation on different environments and preservation conditions from an area belonging to Caatinga, Petrolina, PE. Gaia Sci. 2019 Set;13(1), doi: 10.22478/ufpb.1981-1268.2019v13n1.39803

55. Araújo KCT, Cruz ABS, Fabricante JR. Invasão biológica na Área de Proteção Ambiental Morro do Urubu, Aracaju, Sergipe, Brasil. Rev Biol Ciências da Terra. 2020 Dez;21(1):72-82.

56. Zenni RD. The naturalized flora of Brazil: A step towards identifying future invasive non-native species. Rodriguesia. 2015 Nov;66(4):1137-1144, doi: 10.1590/2175-7860201566413

57. Lowe S, Browne M, Boudjelas S, De Poorter M. 100 of The world worst invasive alien species: A selection from the global invasive species database. Auckland (NZL): The Invasive Species Specialist Group; 2000.

58. Braga CC, Brito JD, Sansigolo CA, Rao TVR. Tempo de resposta da vegetação às variabilidades sazonais da precipitação no Nordeste do Brasil. Rev Bras Agrometeorol. 2003 Mai;11(1):149-157.

59. Becerra JAB, Carvalho S, Ometto J. Relação das sazonalidades da precipitação e da vegetação no bioma caatinga: abordagem multitemporal. In: Anais XVII Simpósio Brasileiro de Sensoriamento RemotoSBSR, João Pessoa-PB, Brasil; 2015. p. 6668-6674.

60. Pérez JE, Nirchio M, Alfonsi C, Muñoz C. The biology of invasions: the genetic adaptation paradox. Biological Invasions. 2006 Mar;8(5):1115-1121, doi: 10.1007/s10530-005-8281-0 


\section{Material Suplementar}

Tabela S1: Código e abreviação das variáveis bioclimáticas obtidas do Worldclim 2 [33], com dados climáticos de 1970-2000 e destaque em sublinhado para as variáveis utilizadas nos modelos baseadas na não correlação entre elas.

\begin{tabular}{ccc}
\hline Código & Variável & Abreviação \\
\hline$\underline{\text { bio1 }}$ & Temperatura média anual & $\underline{\text { TMA }}$ \\
bio2 & Variação da temperatura diurna média & VTDM \\
bio4 & Isotermalidade & ISO \\
$\underline{\text { bio5 }}$ & Sazonalidade da temperatura & SZT \\
bio6 & Temperatura máxima do mês mais quente & $\underline{\text { TMMQ }}$ \\
bio7 & Temperatura mínima do mês mais frio & TMMF \\
bio8 & Amplitude térmica anual & ATA \\
bio9 & Temperatura média do trimestre mais úmido & TMTU \\
bio10 & Temperatura média do trimestre mais seco & TMTS \\
bio11 & Temperatura média do trimestre mais quente & TMTQ \\
$\underline{\text { bio12 }}$ & Temperatura média do trimestre mais frio & TMTF \\
bio13 & Precipitação anual & PA \\
bio14 & Precipitação do mês mais chuvoso & PMMC \\
$\underline{\text { bio15 }}$ & Precipitação do mês mais seco & PMMS \\
bio16 & Sazonalidade da precipitação (coeficiente de variação) & SZP \\
bio17 & Precipitação do trimestre mais chuvoso & PTMC \\
bio18 & Precipitação do trimestre mais seco & PTMS \\
bio19 & Precipitação do trimestre mais quente & PTMQ \\
\hline
\end{tabular}




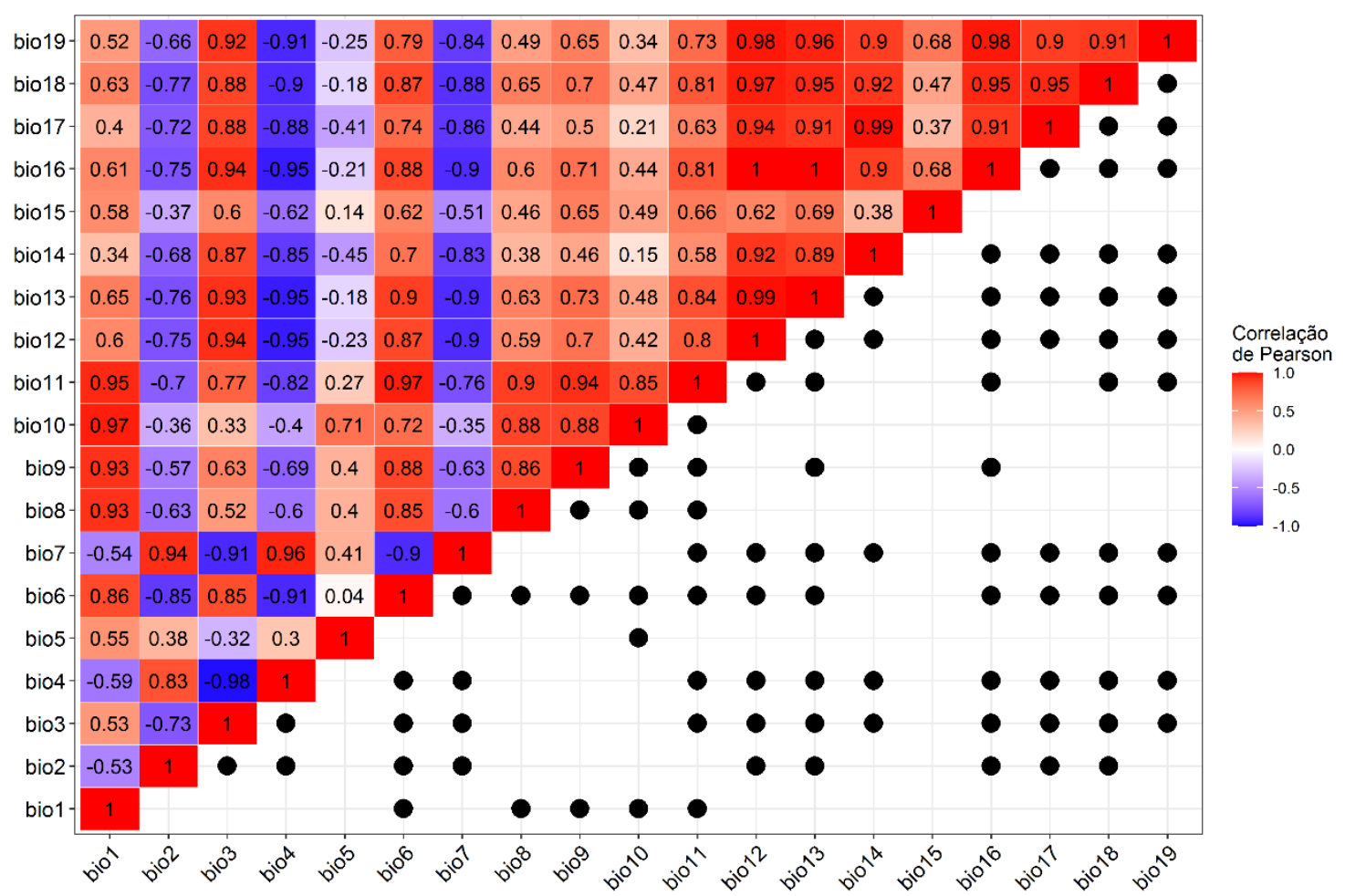

Figura S1: Painel de correlação de Pearson entre todas as variáveis climáticas coletadas do Wordclim 2. Os pontos pretos no triângulo inferior do painel representam a interseç̧ão de variáveis com valores absolutos superiores a 0,7 . 
Tabela S2: Plantas exóticas encontradas em rodovias estaduais e federais do estado de Sergipe e o número de registros em 55 sessões. Nome das famílias e espécies em ordem alfabética.

\begin{tabular}{|c|c|c|}
\hline Família & Espécie & $\begin{array}{c}\mathrm{N}^{\circ} \mathrm{de} \\
\text { registros }\end{array}$ \\
\hline Amaranthaceae & Amaranthus viridis $\mathrm{L}$. & 23 \\
\hline \multirow[t]{3}{*}{ Apocynaceae } & Calotropis procera (Aiton) W.T.Aiton & 23 \\
\hline & Catharanthus roseus (L.) G.Don. & 18 \\
\hline & Cryptostegia madagascariensis Bojer ex Decne. & 3 \\
\hline \multirow[t]{3}{*}{ Asteraceae } & Cosmos caudatus Kunth & 1 \\
\hline & Cosmos sulphureus Cav. & 3 \\
\hline & Tithonia diversifolia (Hemsl.) A.Gray & 2 \\
\hline Combretaceae & Terminalia catappa $\mathrm{L}$ & 38 \\
\hline Crassulaceae & Kalanchoe delagoensis Eckl. \& Zeyh. & 3 \\
\hline \multirow[t]{3}{*}{ Cucurbitaceae } & Cucumis dipsaceus Ehrenb. & 2 \\
\hline & Luffa cylindrica (L.) M.Roem. & 11 \\
\hline & Momordica charantia $\mathrm{L}$. & 20 \\
\hline Cyperaceae & Cyperus rotundus $\mathrm{L}$. & 8 \\
\hline \multirow[t]{2}{*}{ Euphorbiaceae } & Jatropha gossypiifolia $\mathrm{L}$. & 29 \\
\hline & Ricinus communis $\mathrm{L}$. & 46 \\
\hline \multirow[t]{5}{*}{ Fabaceae } & Crotalaria pallida Aiton & 8 \\
\hline & Crotalaria retusa $\mathrm{L}$. & 7 \\
\hline & Leucaena leucocephala (Lam.) de Wit & 51 \\
\hline & Pithecellobium dulce (Roxb.) Benth. & 16 \\
\hline & Prosopis spp. & 19 \\
\hline Meliaceae & Azadirachta indica A. Juss. & 31 \\
\hline Moraceae & Artocarpus heterophyllus Lam. & 18 \\
\hline \multirow[t]{3}{*}{ Myrtaceae } & Eucalyptus spp & 40 \\
\hline & Psidium guajava $\mathrm{L}$. & 17 \\
\hline & Syzygium cumini (L.) Skeels & 11 \\
\hline Nyctaginaceae & Boerhavia diffusa $\mathrm{L}$ & 6 \\
\hline Pinaceae & Pinus caribaea Morelet & 2 \\
\hline \multirow[t]{16}{*}{ Poaceae } & Arundo donax L. & 1 \\
\hline & Cenchrus ciliaris $\mathrm{L}$. & 14 \\
\hline & Cenchrus echinatus $\mathrm{L}$. & 12 \\
\hline & Cenchrus polystachios (L.) Morrone & 16 \\
\hline & Cenchrus purpureus (Schumach.) Morrone & 32 \\
\hline & Cynodon dactylon (L.) Pers. & 32 \\
\hline & Dactyloctenium aegyptium (L.) Willd. & 2 \\
\hline & Digitaria insularis (L.) Fedde & 1 \\
\hline & Eleusine indica (L.) Gaertn. & 6 \\
\hline & Eragrostis ciliaris (L.) R.Br. & 3 \\
\hline & Eragrostis tenella (L.) P.Beauv. ex Roem. \& Schult. & 1 \\
\hline & Megathyrsus maximus (Jacq.) B.K.Simon \& S.W.L.Jacobs & 33 \\
\hline & Melinis minutiflora P.Beauv. & 1 \\
\hline & Melinis repens (Willd.) Zizka & 31 \\
\hline & Sorghum bicolor subsp.arundinaceum (Desv.) de Wet \& J.R Harlan & 9 \\
\hline & Urochloa brizantha (Hochst. ex A. Rich.) R.D.Webster & 29 \\
\hline Polygonaceae & Antigonon leptopus Hook. \& Arn. & 2 \\
\hline \multirow[t]{2}{*}{ Solanaceae } & Nicotiana glauca Graham & 2 \\
\hline & Physalis angulata $\mathrm{L}$. & 2 \\
\hline
\end{tabular}


Tabela S3: Análise de redundância (RDA) com os autovalores e explicação da variância de variáveis preditoras na composição de espécies de plantas exóticas em rodovias de Sergipe.

\begin{tabular}{ccc}
\hline Variável & RDA1 & RDA2 \\
\hline Temperatura média anual (TMA) & $-0,49084$ & $-0,56316$ \\
Temperatura máxima do mês mais quente (TMMQ) & 0,211566 & $-0,64494$ \\
Precipitação anual (PA) & $-0,89722$ & $-0,01691$ \\
Sazonalidade da precipitação (SZP) & $-0,59755$ & $-0,3666$ \\
Eigenvalue & 0,05785 & 0,018071 \\
Explicação (\%) & 11,3236 & 3,537195 \\
\hline $\boldsymbol{p}$ & 0,001 \\
\hline
\end{tabular}


Tabela S4: Resultado do TITAN para as espécies relacionadas em sentido decrescente (z-) e crescente (z+) para espécies de plantas exóticas em resposta as variáveis climáticas registradas nas rodovias do Estado de Sergipe. Apenas espécies que preencheram critérios significativos para $p(\leq 0,05)$, pureza $(\geq 0,95)$ e confiabilidade (Conf. $\geq$ $0,95)$ estão incluídos nesta tabela. Escores de indicadores (IndVal e z) e frequência de ocorrência (Freq.).

\begin{tabular}{|c|c|c|c|c|c|c|c|c|c|c|c|c|}
\hline Variáveis & Espécie & $+/-$ & Obs. & $5 \%$ & $50 \%$ & $95 \%$ & $\mathbf{Z}$ & IndVal & $p$ & Pureza & Conf. & Freq. \\
\hline \multirow{5}{*}{ Temperatura média anual } & A. indica & $\mathrm{Z}-$ & 5,35 & 23,96 & 24,16 & 25,11 & 6,32 & 57,14 & 0,008 & 1,00 & 1,00 & 31 \\
\hline & Eucalyptus spp. & Z- & 4,39 & 24,12 & 24,56 & 25,56 & 4,66 & 57,78 & 0,004 & 0,99 & 0,95 & 40 \\
\hline & M. maximus & Z- & 9,89 & 24,10 & 24,67 & 24,92 & 9,31 & 74,61 & 0,004 & 1,00 & 1,00 & 33 \\
\hline & M. repens & Z- & 5,28 & 24,18 & 24,39 & 24,91 & 5,15 & 55,23 & 0,004 & 0,96 & 0,98 & 31 \\
\hline & U. brizantha & $\mathrm{Z}+$ & 5,77 & 24,65 & 24,92 & 25,24 & 6,23 & 56,25 & 0,004 & 1,00 & 0,99 & 29 \\
\hline \multirow{3}{*}{ Temperatura máxima do mês mais quente } & C. procera & $\mathrm{Z}+$ & 7,13 & 30,60 & 30,98 & 31,03 & 6,82 & 76,67 & 0,004 & 0,99 & 0,99 & 23 \\
\hline & Eucalyptus spp. & $\mathrm{Z}-$ & 3,64 & 30,09 & 30,79 & 31,50 & 4,35 & 59,88 & 0,016 & 0,99 & 0,95 & 40 \\
\hline & M.maximus & Z- & 6,72 & 30,11 & 30,24 & 30,41 & 7,47 & 63,42 & 0,004 & 1,00 & 1,00 & 33 \\
\hline \multirow{6}{*}{ Precipitação anual } & A. indica & $\mathrm{Z}-$ & 7,87 & 811,50 & 959,50 & 1205,50 & 8,16 & 72,22 & 0,004 & 1,00 & 1,00 & 31 \\
\hline & C. procera & Z- & 8,49 & 774,98 & 793,00 & 1169,00 & 9,61 & 69,24 & 0,004 & 1,00 & 1,00 & 23 \\
\hline & M. maximus & $\mathrm{Z}-$ & 7,80 & 943,00 & 1328,00 & 1589,50 & 8,21 & 69,72 & 0,004 & 1,00 & 1,00 & 33 \\
\hline & M. repens & Z- & 7,54 & 1226,50 & 1533,00 & 1563,00 & 8,01 & 73,47 & 0,004 & 1,00 & 1,00 & 31 \\
\hline & Prosopis spp. & Z- & 12,86 & 811,50 & 982,00 & 1062,78 & 12,74 & 84,51 & 0,004 & 1,00 & 1,00 & 19 \\
\hline & U. brizantha & $\mathrm{Z}+$ & 11,26 & 1033,03 & 1214,00 & 1328,00 & 11,61 & 84,09 & 0,004 & 1,00 & 1,00 & 29 \\
\hline \multirow{6}{*}{ Sazonalidade da precipitação } & A. indica & $\mathrm{Z}-$ & 8,54 & 55,74 & 58,10 & 61,63 & 8,85 & 70,42 & 0,004 & 1,00 & 1,00 & 31 \\
\hline & C. roseus & Z- & 5,92 & 57,25 & 58,83 & 61,19 & 6,48 & 47,37 & 0,004 & 1,00 & 1,00 & 18 \\
\hline & J. gossypiifolia & Z- & 4,39 & 55,45 & 62,93 & 64,33 & 4,87 & 63,04 & 0,008 & 0,98 & 0,97 & 29 \\
\hline & M. maximus & Z- & 8,65 & 55,81 & 57,73 & 59,03 & 9,11 & 73,39 & 0,004 & 1,00 & 1,00 & 33 \\
\hline & Prosopis spp. & Z- & 6,80 & 53,99 & 55,81 & 57,82 & 7,69 & 58,98 & 0,004 & 1,00 & 1,00 & 19 \\
\hline & U. brizantha & $\mathrm{z+}$ & 5,18 & 53,92 & 56,05 & 61,20 & 6,11 & 56,25 & 0,004 & 1,00 & 0,99 & 29 \\
\hline
\end{tabular}

